\title{
Involvement of Circadian Clock Gene BMAL1 in Doxorubicin-Induced Inflammation in Vascular Smooth Muscle Cells
}

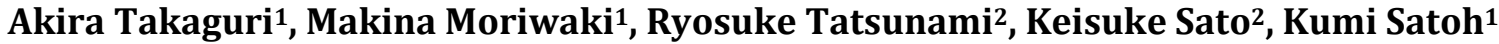 \\ ${ }^{1}$ Department of Pharmacology, Hokkaido University of Science, Hokkaido, Japan \\ ${ }^{2}$ Department of Pharmacy, Hokkaido University of Science, Hokkaido, Japan \\ Email:kumi@hus.ac.jp
}

How to cite this paper: Takaguri, A., Moriwaki, M., Tatsunami, R., Sato, K. and Satoh, K. (2021) Involvement of Circadian Clock Gene BMAL1 in Doxorubicin-Induced Inflammation in Vascular Smooth Muscle Cells. Pharmacology \& Pharmacy, 12, 255268.

https://doi.org/10.4236/pp.2021.1211022

Received: September 26, 2021

Accepted: November 27, 2021

Published: November 30, 2021

Copyright $\odot 2021$ by author(s) and Scientific Research Publishing Inc. This work is licensed under the Creative Commons Attribution International License (CC BY 4.0).

http://creativecommons.org/licenses/by/4.0/

\section{Open Access}

\begin{abstract}
The molecular clock component Brain and Muscle Arnt-Like protein-1 (BMAL1) affects various biologic processes, including cell survival, in numerous cell types. We previously demonstrated that BMAL1 positively regulates cell proliferation in Vascular Smooth Muscle Cells (VSMCs). However, its role in VSMC inflammation remains unelucidated. Because doxorubicin causes phlebitis associated with vascular inflammation, the present study used cultured VSMCs to investigate whether BMAL1 affected doxorubicin-induced vascular inflammation. Doxorubicin treatment led to Increased Interleukin (IL)-6 mRNA expression with an increase in BMAL1 expression in VSMCs. BMAL1 knockdown significantly increased IL-6 mRNA and further enhanced doxorubicin-induced IL-6 mRNA expression in VSMCs. BMAL1 knockdown also significantly decreased cell viability and affected the expression of other clock genes, including Per1 and Clock. Furthermore, the levels of nuclear factor erythroid 2-related factor 2, which has anti-inflammatory effects, increased in VSMCs with BMAL1 knockdown. Finally, BMAL1 knockdown increased NADPH oxidase 4 mRNA, p38 $\alpha$ mRNA, and p38 $\beta$ mRNA levels, leading to increased total p38 Mitogen-Activated Protein Kinase (MAPK) and phosphorylated p38 MAPK. IL-6 mRNA induction caused by BMAL1 knockdown was significantly inhibited in VSMCs following pretreatment with SB203580, a p38 MAPK inhibitor. Our findings demonstrated that decreased BMAL1 expression caused VSMC inflammation via p38 MAPK activation. Moreover, doxorubicin-induced inflammation in VSMCs was further enhanced when BMAL1 expression levels were low. Thus, BMAL1 may be a novel therapeutic target to treat inflammatory disease, including doxorubicin-induced phlebitis.
\end{abstract}

\section{Keywords}

BMAL1, Doxorubicin, IL-6, MAPK, p38, VSMC 


\section{Introduction}

Doxorubicin is a highly potent, effective broad-spectrum anticancer chemotherapeutic drug used to treat various cancers, including solid and hematologic tumors. However, because it elicits dose-dependent toxic side effects associated with cardiotoxicity, leading to dilated cardiomyopathy and heart failure [1] [2], its clinical use is limited. Additionally, although the frequency of side effects is relatively low compared with that of cardiotoxicity, doxorubicin also causes systematic inflammation, including phlebitis [3] [4]. Phlebitis is characterized by an inflammatory response associated with elevated interleukin (IL)-6 expression, causing various forms of tissue damage and symptoms of pain and warmth [5] [6].

Clock genes form transcription-translational feedback loops that maintain circadian rhythms [7] [8] [9]. Brain and Muscle Arnt-Like protein-1 (BMAL1) is a crucial circadian clock gene and the only Clock gene where deletion completely ablates all mammalian rhythms [10]. BMAL1 forms a heterodimer with a clock circadian regulator (CLOCK) and its heterodimers, which bind to the E-box element, increasing the expression of genes such as PER and CRY, which code for the Period Circadian Regulator (PER) and Cryptochrome Circadian Regulator (CRY), respectively. PER/CRY heterodimers inhibit BMAL1/CLOCK-dependent gene transcription. Additionally, the heterodimeric CLOCK: BMAL1 complex accelerates transcription of the DNA-binding orphan nuclear receptor reverse erythroblastosis virus (REV-ERB)- $\alpha / \beta$ and retinoid-related orphan receptor (ROR)- $\alpha,-\beta,-\gamma$. RORs activate the transcriptional expression of BMAL1, whereas REV-ERBs repress it by binding to the ROR element [11] [12].

Circadian rhythm disruption increases the risk of various diseases, including metabolic disorders and cardiovascular diseases [13] [14]. BMAL1 was recently reported to affect a diverse range of biologic processes, including signal transduction associated with apoptosis, cell proliferation, and cell differentiation, in many cell types. Furthermore, accumulating evidence suggests that BMAL1 is crucial in the pathophysiology of vascular disorders, including hypertension and abdominal aortic aneurysms [15] [16] [17]. We recently reported that BMAL1 positively regulates Vascular Smooth Muscle Cell (VSMC) proliferation [18]. Thus, although BMAL1 regulates a variety of vascular functions, its role in VSMC inflammation remains largely unelucidated. Therefore, this study aimed to determine whether BMAL1 affects inflammation associated with doxorubicin-induced phlebitis in VSMCs.

\section{Materials and Method}

\subsection{Drugs and Reagents}

Dulbecco's Modified Eagle's Medium (DMEM), doxorubicin, Sodium Dodecyl Sulfate (SDS), bovine serum albumin, protease inhibitor cocktail, and anti-GAPDH (014-25524) were purchased from FUJIFILM Wako Pure Chemical Corporation (Osaka, Japan). SB203580 was purchased from Cayman Chemical (Ann Arbor, 
MI, USA), fetal bovine serum from Biowest (Nuaillé, France), and Pierce ECL Western Blotting Substrate from Thermo Scientific (Rockford, IL, USA). Anti-phospho-p38 MAPK (\#9211), anti-p38 MAPK (\#9212), Horseradish Peroxidase (HRP)linked-anti rabbit IgG (\#7074), and HRP-linked anti-mouse IgG (\#7076) were purchased from Cell Signaling Technology (Beverly, MA, USA). Anti-BMAL1 (sc365645) was purchased from Santa Cruz Biotechnology (Santa Cruz, CA, USA) and anti-Nrf2 (\#16396-1-AP) from Proteintech (Rosemont, IL, USA). Control short interfering RNA (siRNA) was obtained from Sigma (St. Louis, MO, USA). BMAL1 siRNA and Lipofectamine RNAiMAX Transfection Reagent were obtained from Invitrogen (Carlsbad, CA, USA). FastGene RNA Basic Kits were purchased from Nippon Genetics (Tokyo, Japan). PrimeScript RT Reagent Kits and TB Green Premix Ex Taq II were purchased from TaKaRa Bio Inc. (Shiga, Japan).

\subsection{Cell Culture and siRNA Transfection}

The experimental plan was approved by the president of Hokkaido University of Science (No. 2021-007) and was confirmed to be consistent with the Guiding Principles for the Care and Use of Experimental Animals at the Hokkaido University of Science. 5-6-week-old male Sprague Dawley rats were anesthetized with $0.75 \mathrm{mg} / \mathrm{kg}$ medetomidine hydrochloride (Domitor ${ }^{\circledR}$; Nippon Zenyaku Kogyo Co., Ltd., Fukushima, Japan), 4.0 mg/kg midazolam (Dormicum; ${ }^{\circledR}$ Astellas Pharma Inc., Tokyo, Japan) and $5.0 \mathrm{mg} / \mathrm{kg}$ butorphanol (Vetorphale ${ }^{\oplus}$; Meiji Seika Pharma Co., Ltd., Tokyo, Japan) before dissection. VSMCs isolated from thoracic aortas using an enzymatic digestion method were cultured in DMEM containing $10 \%$ fetal bovine serum, $100 \mathrm{U} / \mathrm{mL}$ penicillin, and $100 \mu \mathrm{g} / \mathrm{mL}$ streptomycin, as previously described [18]. When confluency of approximately $80 \%-90 \%$ was reached, the cells were transfected with $50 \mathrm{nM}$ siRNA using Lipofectamine RNAiMAX Transfection Reagent, according to the manufacturer's protocol.

\subsection{Cell Viability Assay}

After treating the VSMCs with doxorubicin at the indicated concentrations or with siRNA targeting BMAL1, cell viability was assessed using cell counting kit-8 (Dojindo Molecular Technologies, Kumamoto, Japan).

\subsection{Western Blot}

After treatment, cell lysis and subsequent western blots were performed, as previously described [18].

\subsection{Reverse Transcription-Quantitative Polymerase Chain Reaction (qPCR) Analysis}

Total RNA extraction, cDNA synthesis, and subsequent qPCR were performed, as previously described [18]. The primers used are presented in Table 1. GAPDH was used as the housekeeping (control) gene. 
Table 1. Details of the primers used in our study.

\begin{tabular}{|c|c|c|}
\hline Target gene & Primer direction & Primer sequence \\
\hline \multirow{2}{*}{ GAPDH } & Forward & 5'-GGCACAGTCAAGGCTGAGAATG-3' \\
\hline & Reverse & 5'-ATGGTGGTGAAGACGCCAGTA-3' \\
\hline \multirow{2}{*}{ BMAL1 } & Forward & 5'-TTCATGAACCCGTGGACCAA-3' \\
\hline & Reverse & 5'-CCCTGGAATGCCTGGAACA-3' \\
\hline \multirow{2}{*}{ CLOCK } & Forward & 5'-TGCCAGCTCATGAGAAGATG-3' \\
\hline & Reverse & 5'-CATCGCTGGCTGTGTTAATG-3' \\
\hline \multirow{2}{*}{ PER1 } & Forward & 5'-GCCTCAGGCCCTCGATGTAA-3' \\
\hline & Reverse & 5'-CGAGTGGCCAGGATCTTGAA-3' \\
\hline \multirow{2}{*}{ PER2 } & Forward & 5'-CACCCTGAAAAGAAAGTGCGA-3' \\
\hline & Reverse & 5'-CAACGCCAAGGAGCTCAAGT-3' \\
\hline \multirow{2}{*}{ Mapk11 } & Forward & 5'-CCCAGCAATGTAGCAGTGAATGA-3' \\
\hline & Reverse & 5'-CCATGATGCAGCCCACAGA-3' \\
\hline \multirow{2}{*}{ Mapk14 } & Forward & 5'-ATGGGTGCATGTGTGCATGA-3' \\
\hline & Reverse & 5'-CTACTGATGGCAGGAGCCTGTG-3' \\
\hline \multirow{2}{*}{ Nox1 } & Forward & 5'-GGAGACCAATGTGGGACAATGA-3' \\
\hline & Reverse & 5'-CTTGAGTACCGCCGACAGCA-3' \\
\hline \multirow{2}{*}{ Nox 4} & Forward & 5'-ACTGCCTCCATCAAGCCAAGA-3' \\
\hline & Reverse & 5'-GACTTCCAAATGGGCCATCAA-3' \\
\hline \multirow{2}{*}{ IL6 } & Forward & 5'-ATTGTATGAACAGCGATGATGCAC-3' \\
\hline & Reverse & 5'-CCAGGTAGAAACGGAACTCCAGA-3' \\
\hline \multirow{2}{*}{ Nrf2 } & Forward & 5'-GCTGCCATTAGTCAGTCGCTCTC-3' \\
\hline & Reverse & 5'-ACCGTGCCTTCAGTGTGCTTC-3' \\
\hline \multirow{2}{*}{ Hmox1 } & Forward & 5'-AGGTGCACATCCGTGCAGAG-3' \\
\hline & Reverse & 5'-CTTCCAGGGCCGTATAGATATGGTA-3' \\
\hline
\end{tabular}

GAPDH was used as the housekeeping (control) gene.

\section{Statistical Analysis of Data}

Data were expressed as means \pm Standard Error of the Mean (SEM). Differences between the groups were evaluated using the Student $t$-test. GraphPad Prism, version 6 (GraphPad Software, San Diego, CA, USA) was used for all statistical analyses. $P$ values $<0.05$ were considered statistically significant.

\section{Results}

\subsection{Doxorubicin Increases IL-6 Induction and BMAL1 Expression in VSMCs}

We tested whether doxorubicin affected cell viability and IL-6 mRNA expression in VSMCs. Doxorubicin treatment significantly increased IL-6 mRNA expres- 
sion without affecting cytotoxicity (Figure 1(a) and Figure 1(b)), suggesting that doxorubicin causes inflammation by upregulating IL-6 expression in VSMCs. Next, we investigated the effect of doxorubicin treatment on BMAL1 expression in VSMCs. BMAL1 mRNA (Figure 1(c)) and BMAL1 protein (Figure 1(d)) expression were significantly increased following the treatment, indicating that doxorubicin modulated the expression of clock gene BMAL1 at a transcriptional level in VSMCs.

\subsection{BMAL1 Knockdown Further Increases Doxorubicin-Induced IL-6 Induction in VSMCs}

We assessed doxorubicin-induced IL-6 mRNA expression in VSMCs with BMAL1 knockdown to determine the role of BMAL1 in doxorubicin-induced inflammation. The VSMCs treated with $50 \mathrm{nM}$ siRNA targeting BMAL1 showed significantly decreased BMAL1 mRNA (Figure 2(a)) and BMAL1 protein (Figure 2(b)) expression. Interestingly, BMAL1 knockdown significantly reduced cell viability

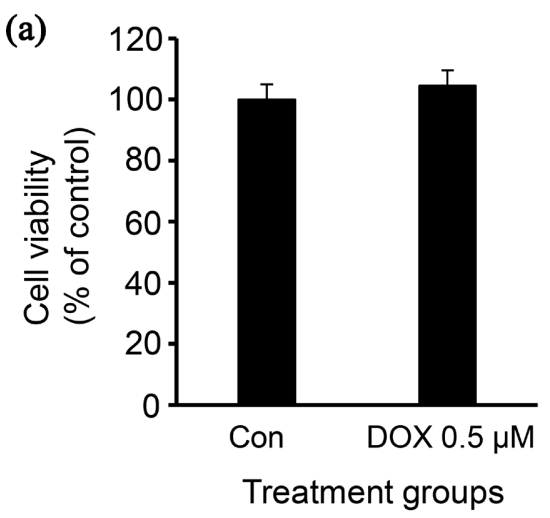

(c)

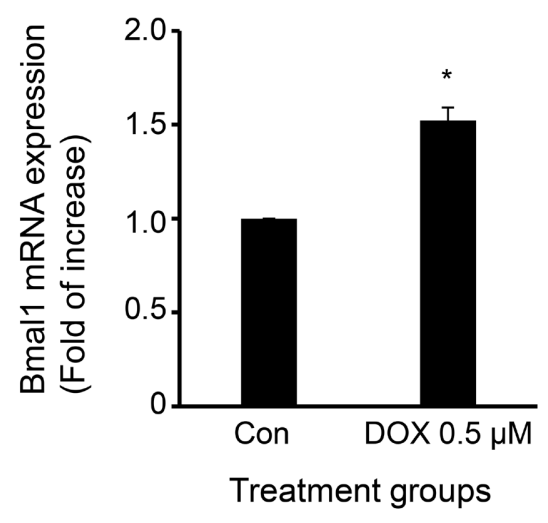

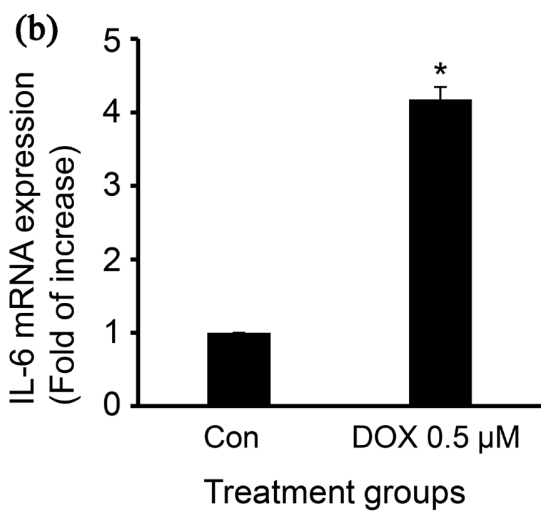

(d)

IB: Bmal1

IB: GAPDH
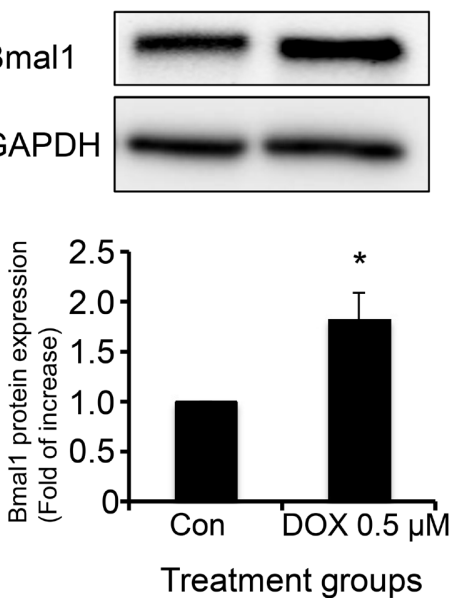

Figure 1. Doxorubicin (DOX) increases IL-6 and BMAL1 expression in cultured VSMCs. The cells were treated with $0.5 \mu \mathrm{M}$ DOX for $6 \mathrm{~h}$. Cell viability was determined using cell counting kit-8 (a). IL-6 mRNA (b) and BMAL1 mRNA (c) expression were evaluated using qPCR. The samples were then subjected to SDS-polyacrylamide gel electrophoresis and immunoblotted using the indicated antibodies (d). Data are presented as means \pm SEM of three experiments. ${ }^{\star} P<0.05$ versus control siRNA-transfected cells. Con, control; IB, immunoblot. 
(a)

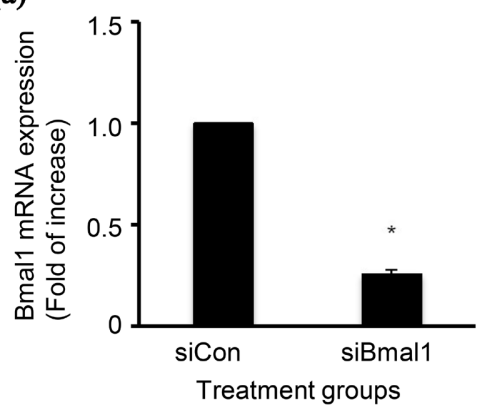

(d)

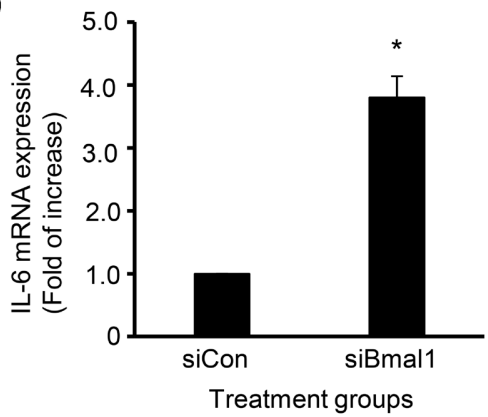

(g)

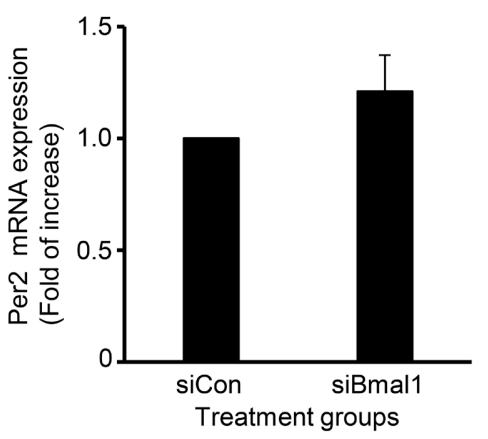

(b)

IB: Bmal1

IB: GAPDH
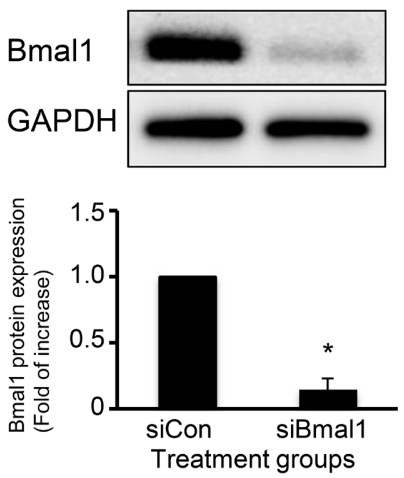

(e)

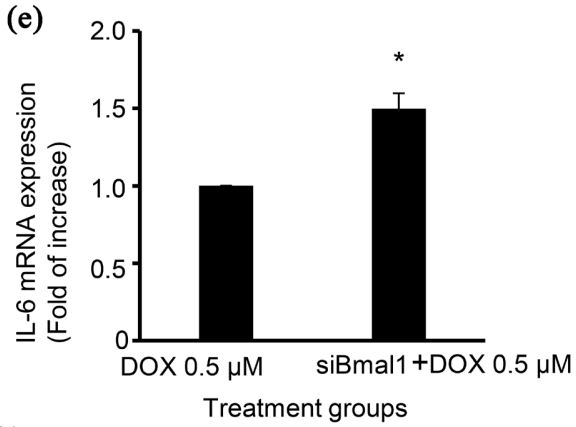

(h)

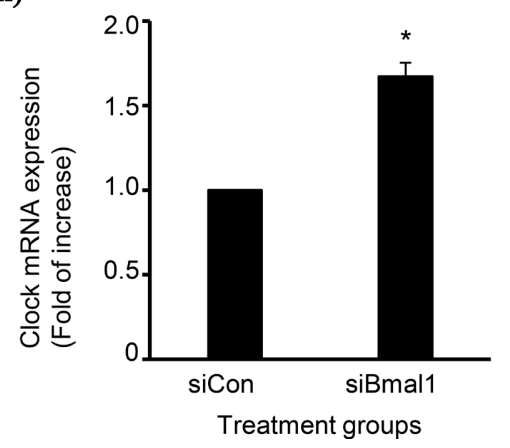

(c)

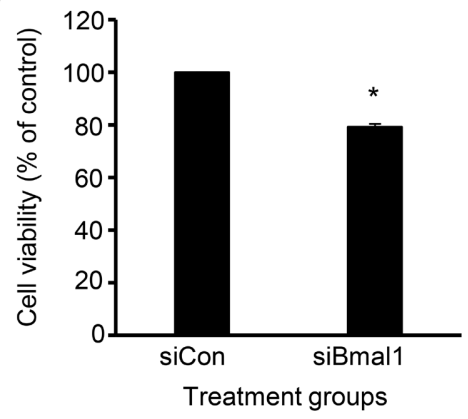

(f)

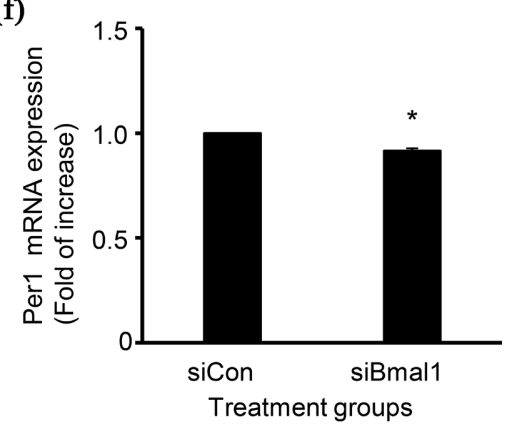

Figure 2. BMAL1 knockdown further increases doxorubicin (DOX)-induced IL-6 mRNA expression with decreased cell viability in VSMCs. The cells were transfected with $50 \mathrm{nM}$ of siRNA targeting BMAL1 or with siRNA control (siCon). BMAL1 mRNA expression was evaluated using qPCR (a). The samples were then subjected to SDS-polyacrylamide gel electrophoresis and immunoblotted using the indicated antibodies (b). Cell viability was determined using cell counting kit-8 assay (c). IL-6, Per1, Per2, and CLOCK mRNA expression were evaluated using $\mathrm{qPCR}(\mathrm{d})-(\mathrm{g})$. The cells were transfected with siRNA targeting BMAL1 or with siCon and then treated with $0.5 \mu \mathrm{M}$ DOX for $6 \mathrm{~h}$. IL- 6 mRNA expression was evaluated using qPCR (h). Data are presented as means \pm SEM of three experiments. ${ }^{*} P<0.05$ versus control siRNA-transfected cells. IB, immunoblot.

(Figure 2(c)) and increased IL-6 mRNA expression (Figure 2(d)). BMAL1 knockdown further increased doxorubicin-induced IL-6 mRNA expression (Figure 2(c)), suggesting that BMAL1 played a protective role in doxorubicin-induced inflammation in VSMCs. Additionally, we tested whether BMAL1 knockdown affected other clock genes in VSMCs. BMAL1 knockdown significantly decreased Per1 mRNA expression (Figure 2(f)) and significantly increased CLOCK mRNA expression (Figure 2(h)). On the other hand, the BMAL1 knockdown did not alter Per2 mRNA expression in VSMCs (Figure 2(g)). These findings suggested that other clock genes might be involved in increased IL-6 expression in VSMCs with BMAL1 knockdown. 


\subsection{BMAL1 Knockdown Increases Nuclear Factor Erythroid 2-Related Factor 2 (Nrf2) Expression in VSMCs}

Nrf2 exerts an anti-inflammatory response by upregulating antioxidant genes. Therefore, we examined whether BMAL1 knockdown affected Nrf2 expression in VSMCs. We found that BMAL1 knockdown of significantly increased Nrf2 mRNA (Figure 3(a)) and protein (Figure 3(b)) expression in VSMCs. Additionally, Heme Oxygenase-1 (HO-1), a target gene of Nrf2, was significantly increased VSMCs with BMAL1 knockdown (Figure 3(c)). These results suggested that Nrf2 protects against inflammation induced by BMAL1 knockdown in VSMCs.

\section{4. p38 Mitogen-Activated Protein Kinase (MAPK) is Involved in the Induction of IL-6 mRNA Expression in VSMCs with BMAL1 Knockdown}

The Reactive Oxygen Species (ROS)/p38 MAPK pathway is crucial for the induction of IL-6 expression in VSMCs. Therefore, we first examined the effect of BMAL1 knockdown on NADPH Oxidase (Nox) 1 and Nox4, which are involved in ROS production and p38 MAPK activation. BMAL1 knockdown significantly increased Nox4 but not Nox1 mRNA expression in VSMCs (Figure 4(a) and Figure 4(b)). Additionally, BMAL1 knockdown significantly increased the phosphorylated p38 MAPK in VSMCs Figure 4(c)). Interestingly, BMAL1 knockdown also markedly increased total p38 MAPK protein expression and p38 MAPK mRNA (Figures 4(c)-(e)), indicating that BMAL1 knockdown increased p38 MAPK activation accompanied by $\mathrm{p} 38 \alpha$ and p38 $\beta$ mRNA upregulation in VSMCs. Increased IL-6 mRNA expression due to BMAL1 knockdown was significantly inhibited following pretreatment with SB203580, a p38 MAPK inhibitor (Figure 4(f)). These results suggest that p38 MAPK activation played a critical role in increased IL-6 mRNA expression VSMCs with BMAL1 knockdown.

(a)

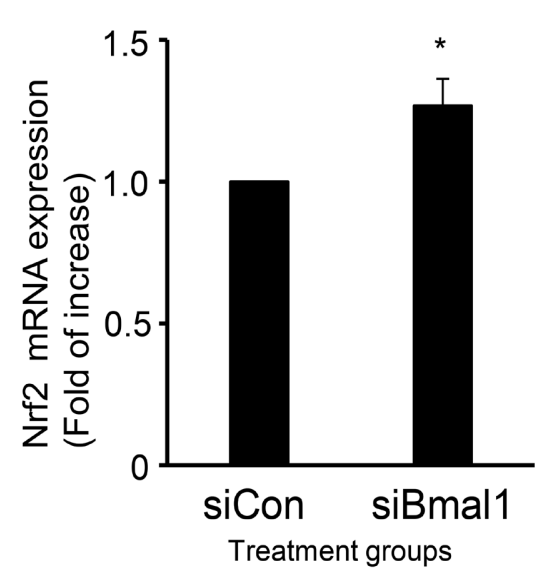

(b)

IB: Nrf2

IB: GAPDH
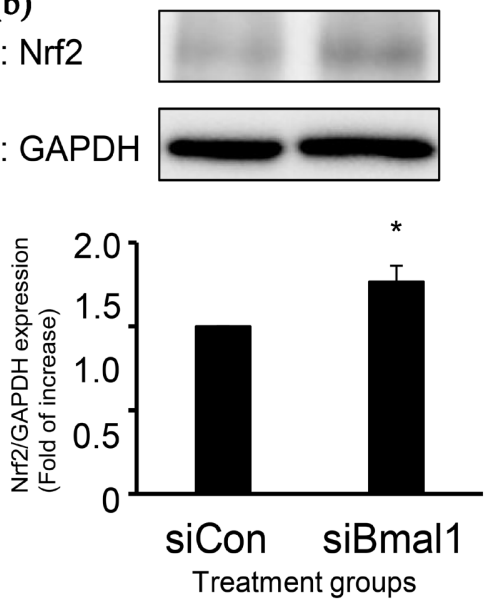

(c)

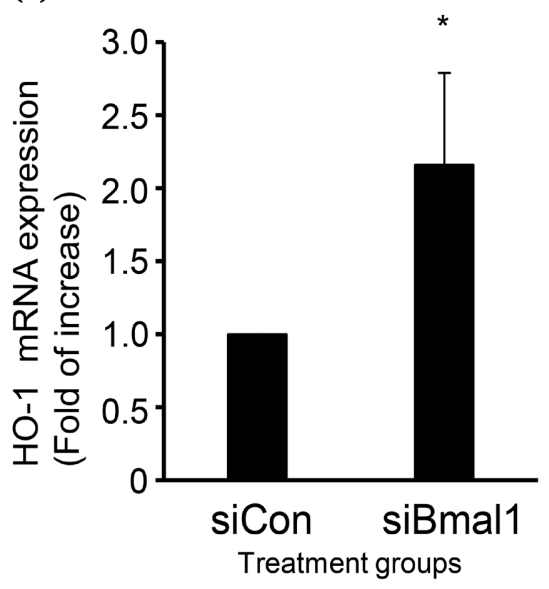

Figure 3. BMAL1 knockdown increases Nrf2 mRNA and protein expression in VSMCs. The cells were transfected with $50 \mathrm{nM}$ of siRNA targeting BMAL1 or with control siRNA (siCon). Nrf2 mRNA (a) and HO-1 (c) expression were evaluated using qPCR. The samples were then subjected to SDS-PAGE and immunoblotted using the indicated antibodies (b). Data are presented as means \pm SEM of three experiments. ${ }^{\star} P<0.05$ versus control-siRNA transfected cells. 
(a)

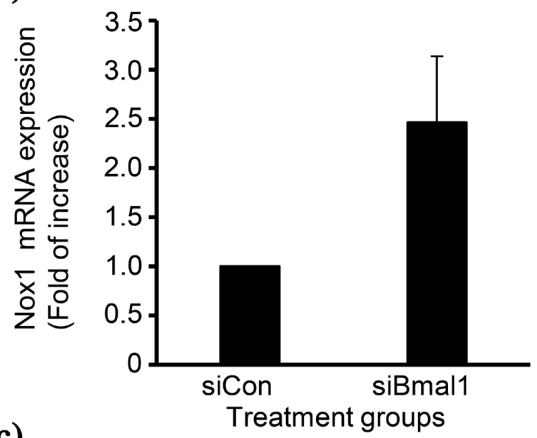

(c)

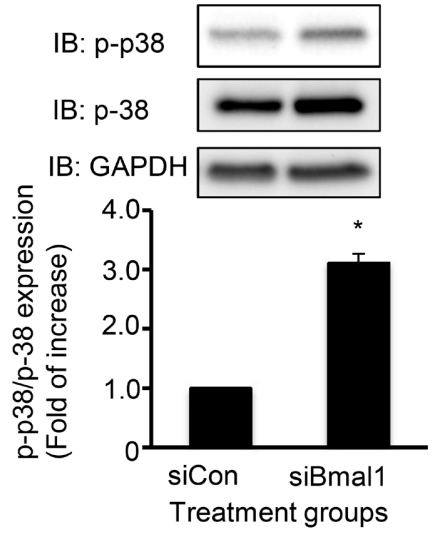

(e)

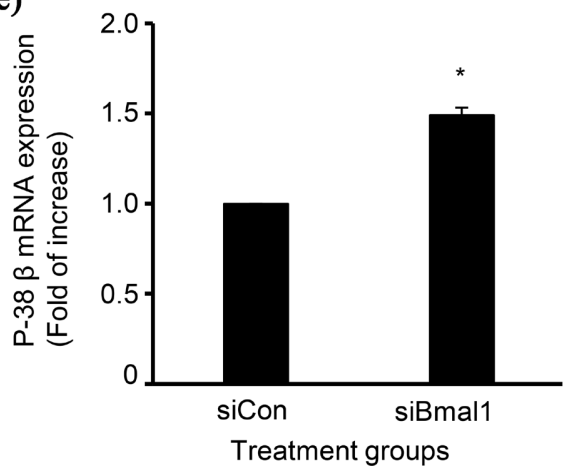

(b)

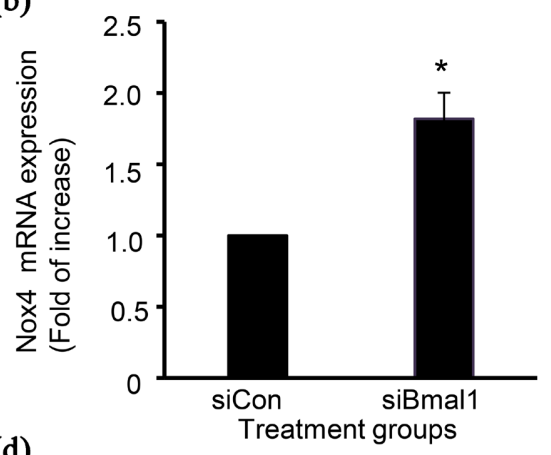

d)

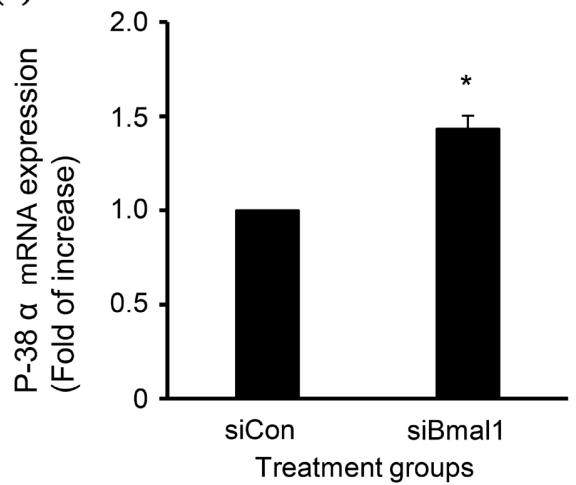

(f)

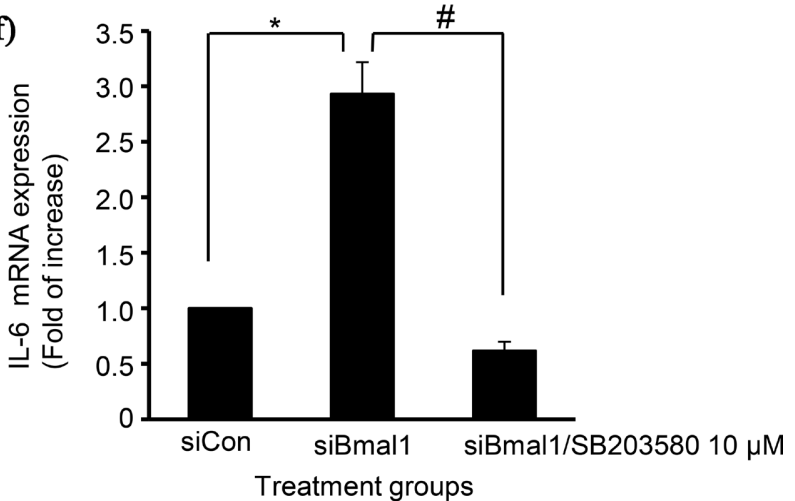

Figure 4. BMAL1 knockdown increases IL-6 mRNA expression by p38 upregulation and activation in VSMCs. The cells were transfected with $50 \mathrm{nM}$ of siRNA targeting BMAL1 or with control siRNA (siCon). Nox1 (a), Nox4 (b), p38 $\alpha$ (d), and p38 $\beta$ (e) expression were evaluated using qPCR. The samples were then subjected to SDS-polyacrylamide gel electrophoresis and immunoblotted using the indicated antibodies (c). The cells were transfected with $50 \mathrm{nM}$ of siRNA targeting BMAL1 or with siCon and then treated with $10 \mu \mathrm{M}$ SB203580. IL-6 mRNA expression was evaluated using qPCR (f). Data are presented as means \pm SEM of three experiments. ${ }^{*} P<0.05$ versus control siRNA-transfected cells. ${ }^{\#} P<0.05$ versus BMAL1 siRNA-transfected cells.

\section{Discussion}

Although clock gene proteins were recently recognized as signal molecules involved in a variety of cell responses, including proliferation and apoptosis [18] [19] [20], the role of BMAL1 in vascular inflammation is unclear. In the present study, we showed that doxorubicin increased BMAL1 mRNA and protein expression with elevated IL-6 mRNA expression in VSMCs. We also demonstrated that BMAL1 knockdown further increased doxorubicin-induced IL-6 mRNA 
expression and that IL-6 induction caused by BMAL1 knockdown was partially mediated by p38 MAPK activation in VSMCs.

Vascular inflammation is caused by various inflammatory cytokines produced by VSMCs and macrophage expressed with alkaline phosphatase [21]. Among these cytokines, IL-6 plays a crucial role in vascular inflammation, leading to various cardiovascular diseases, including phlebitis and atherosclerosis [22] [23] [24]. IL-6 induction in VSMCs is triggered by a variety of stimuli, such as angiotensin II and superoxide [25] [26]. Our results revealed that doxorubicin increased IL- 6 mRNA and protein expression, suggesting that doxorubicin induces inflammation, at least in part, through the induction of IL-6 in VSMCs. Consistent with our observation that doxorubicin increases BMAL1 expression, the anticancer drug paclitaxel upregulates BMAL1 expression in mouse kidney and cultured human HK-2 renal cells [27], suggesting it affects the expression of clock gene BMAL1 in a variety of cell types. Further studies are necessary to elucidate the mechanisms whereby doxorubicin affects BMAL1 expression in VSMCs.

It remains unclear whether BMAL1 affects doxorubicin-induced inflammation in VSMCs. We demonstrated that the BMAL1 knockdown increased doxorubicin-induced IL-6 mRNA expression, suggesting that doxorubicin-induced BMAL1 negatively regulates doxorubicin-induced inflammation in VSMCs. In support of our findings, cardiomyocyte-specific BMAL1 deletion in mice was reported to induce proinflammatory cytokines [28], and BMAL1 deletion in macrophages was found to further increase lipopolysaccharide-induced IL- $1 \beta$ mRNA expression [29]. Additionally, given that BMAL1 expression is relatively low at midnight compared with daytime [30], it should be noted that doxorubicin might cause vascular pain and phlebitis more easily when BMAL1 levels are low.

BMAL1 deletion affects the expression of other clock genes and causes clock dysfunction [10]. We found that BMAL1 knockdown reduced Per1 and increased CLOCK in VSMCs. Per1 has been reported to increase IL-6 mRNA expression in primary spinal astrocytes [31], suggesting that decreased Per1 expression caused by BMAL1 knockdown partially contributes to the induction of IL-6 mRNA expression in VSMCs. Further investigations are necessary to determine whether other clock genes are involved in the mechanism of VSMC inflammation.

Nrf2 is a transcriptional factor that protects against inflammation and oxidative stress through the induction of $\mathrm{HO}-1$ and directly binds to the promoter region of IL- 6 to suppress IL- 6 mRNA expression in bone marrow-derived macrophages [32]. Therefore, we investigated whether BMAL1 regulates inflammation by controlling Nrf2 expression in VSMCs. We found that BMAL1 knockdown significantly increased Nrf2 mRNA and protein expression, followed by increased HO-1 expression, the target gene of Nrf2 in VSMCs. In contrast, $B M A L 1$ deletion was reported to decrease Nrf2 expression, which induces proinflammatory cytokines in macrophages [29], suggesting that the role of BMAL1 in Nrf2 expression differs among cell types.

p38 MAPK belongs to the MAPK kinase family, which regulates a variety of cellular responses, including apoptosis and inflammation [33] [34]. P38 MAPK 
is activated by ROS-dependent mechanisms in VSMCs and other cells [35] [36]. The increased expression of Nox family proteins, which are catalytic subunits of $\mathrm{NAD}(\mathrm{P}) \mathrm{H}$ oxidases, leads to ROS production in VSMCs. Among the Nox family members, Nox1 and Nox4 are expressed at relatively higher levels than Nox2 and Nox3 in VSMCs [37]. We found that Nox4, but not Nox 1, was significantly increased and that 38 MAPK phosphorylation was also significantly increased following BMAL1 knockdown in VSMCs, indicating that increased Nox4 activates p38 MAPK in VSMCs. Upregulated Nox4 expression was reported as associated with increased p38 MAPK phosphorylation in inflammation sites in atherosclerotic regions [38].

Increased IL- 6 mRNA induced by BMAL1 knockdown was inhibited by pretreatment with a p38 MAPK inhibitor, indicating that p38 MAPK activation is crucial in the induction of IL- 6 by BMAL1 knockdown in VSMCs. Interestingly, total p38 MAPK was also increased by BMAL1 knockdown through the upregulation of p38 MAPK mRNA expression in VSMCs. In support of our results, MAPK activity was reported to exhibit a circadian rhythm peak in the mid to late subjective night in various clock structures [39] [40]. This suggests that circadian clock genes affect MAPK expression and functions. In fact, several circadian clock genes exert a variety of biologic functions through the p38 MAPK signaling pathway [31] [41] [42].

\section{Conclusion}

Doxorubicin induces inflammation with increased BMAL1 expression in VSMCs. BMAL1 knockdown increases IL-6 induction, which is partially mediated by p38 MAPK activations in VSMCs. Our findings suggest that decreased BMAL1 expression strengthens inflammation induced by doxorubicin.

\section{Funding}

This study was supported, in part, by JSPS KAKENHI [grant number 18K06703].

\section{Conflicts of Interest}

The authors declare no conflicts of interest regarding the publication of this paper.

\section{References}

[1] Ferreira, A.L., Matsubara, L.S. and Matsubara, B.B. (2008) Anthracycline-Induced Cardiotoxicity. Cardiovascular and Hematological Agents in Medicinal Chemistry, 6, 278-281. http://doi.org/10.2174/187152508785909474

[2] Roca-Alonso, L., Pellegrino, L., Castellano, L. and Stebbing, J. (2012) Breast Cancer Treatment and Adverse Cardiac Events: What Are the Molecular Mechanisms? Cardiology, 122, 253-259. https://doi.org/10.1159/000339858

[3] Leal, A.D., Kadakia, K.C., Looker, S., Hilger, C., Sorgatz, K., Anderson, K. and Loprinzi, C.L. (2014) Fosaprepitant-Induced Phlebitis: A Focus on Patients Receiving Doxorubicin/Cyclophosphamide Therapy. Supportive Care in Cancer, 22, 1313-1317. 
https://doi.org/10.1007/s00520-013-2089-8

[4] Wang, L., Chen, Q., Qi, H., Wang, C., Wang, C., Zhang, J. and Dong, L. (2016) Doxorubicin-Induced Systemic Inflammation is Driven by Upregulation of Toll-Like Receptor TLR4 and Endotoxin Leakage. Cancer Research, 76, 6631-6642. https://doi.org/10.1158/0008-5472.CAN-15-3034

[5] Kim, K., Kim, J.H., Kim, I., Seong, S. and Kim, N. (2020) Rev-erb $\alpha$ Negatively Regulates Osteoclast and Osteoblast Differentiation through p38 MAPK Signaling Pathway. Molecules and Cells, 43, 34-47. https://doi.org/10.14348/molcells.2019.0232

[6] Liu, L., Su, S.W., Zhou, P., Song, R. and Sun, H.Y. (2017) External Application of Moisture Exposed Burn Ointment for Phlebitis: A Meta-Analysis of Randomized Controlled Trials. International Journal of Medicine and Medicine Sciences, 9, 158-173. https://doi.org/10.5897/IJMMS2017.1310

[7] Bugge, A., Feng, D., Everett, L.J., Briggs, E.R., Mullican, S.E., Wang, F. and Lazar, M.A. (2012) Rev-erb $\alpha$ and Rev-erb $\beta$ Coordinately Protect the Circadian Clock and Normal Metabolic Function. Genes and Development, 26, 657-667. https://doi.org/10.1101/gad.186858.112

[8] Cho, H., Zhao, X., Hatori, M., Yu, R.T., Barish, G.D., Lam, M.T. and Evans, R.M. (2012) Regulation of Circadian Behaviour and Metabolism by REV-ERB- $\alpha$ and REVERB- $\beta$. Nature, 485, 123-127. https://doi.org/10.1038/nature11048

[9] Gallego, M. and Virshup, D. M. (2007) Post-Translational Modifications Regulate the Ticking of the Circadian Clock. Nature Reviews. Molecular Cell Biology, 8, 139-148. https://doi.org/10.1038/nrm2106

[10] Bunger, M.K., Wilsbacher, L.D., Moran, S.M., Clendenin, C., Radcliffe, L.A., Hogenesch, J.B. and Bradfield, C.A. (2000) Mop3 Is an Essential Component of the Master Circadian Pacemaker in Mammals. Cell, 103, 1009-1017.

https://doi.org/10.1016/S0092-8674(00)00205-1

[11] Preitner, N., Damiola, F., Lopez-Molina, L., Zakany, J., Duboule, D., Albrecht, U. and Schibler, U. (2002) The Orphan Nuclear Receptor REV-ERB $\alpha$ Controls Circadian Transcription within the Positive Limb of the Mammalian Circadian Oscillator. Cell, 110, 251-260. https://doi.org/10.1038/s441598-017-02907-6

[12] Orozco-Solis, R. and Sassone-Corsi, P. (2014) Epigenetic Control and the Circadian Clock: Linking Metabolism to Neuronal Responses. Neuroscience, 264, 76-87. https://doi.org/10.1016/j.neuroscience.2014.01.043

[13] Brum, M.C., Filho, F.F., Schnorr, C.C., Bottega, G.B. and Rodrigues, T.C. (2015) Shift Work and Its Association with Metabolic Disorders. Diabetology and Metabolic Syndrome, 7, Article No. 45. https://doi.org/10.1186/s13098-015-0041-4

[14] Skogstad, M., Mamen, A., Lunde, L.K., Ulvestad, B., Matre, D., Aass, H.C. and Sirnes, P.A. (2019) Shift Work Including Night Work and Long Working Hours in Industrial Plants Increases the Risk of Atherosclerosis. International Journal of Environmental Research and Public Health, 16, Article No. 521. https://doi.org/10.3390/ijerph16030521

[15] Lutshumba, J., Liu, S., Zhong, Y., Hou, T., Daugherty, A., Lu, H. and Gong, M.C. (2018) Deletion of BMAL1 in Smooth Muscle Cells Protects Mice from Abdominal Aortic Aneurysms. Arteriosclerosis, Thrombosis, and Vascular Biology, 38, 1063-1075. https://doi.org/10.1161/ATVBAHA.117.310153

[16] Yang, G., Zhang, J., Jiang, T., Monslow, J., Tang, S.Y., Todd, L. and FitzGerald, G.A. (2020) Bmal1 Deletion in Myeloid Cells Attenuates Atherosclerotic Lesion Development and Restrains Abdominal Aortic Aneurysm Formation in Hyperlipidemic 
Mice. Arteriosclerosis, Thrombosis, and Vascular Biology, 40, 1523-1532. https://doi.org/10.1161/ATVBAHA.120.314318

[17] Woon, P.Y., Kaisaki, P.J., Bragança, J., Bihoreau, M.T., Levy, J.C., Farrall, M. and Gauguier, D. (2007) Aryl Hydrocarbon Receptor Nuclear Translocator-Like (BMAL1) Is Associated with Susceptibility to Hypertension and Type 2 Diabetes. Proceedings of the National Academy of Sciences of the United States of America, 104, 14412-14417. https://doi.org/10.1073/pnas.0703247104

[18] Takaguri, A., Sasano, J., Akihiro, O. and Satoh, K. (2020) The Role of Circadian Clock Gene BMAL1 in Vascular Proliferation. European Journal of Pharmacology, 872, Article ID: 172924. https://doi.org/10.1016/j.ejphar.2020.172924

[19] Ma, Z., Jin, X., Qian, Z., Li, F., Xu, M., Zhang, Y. and Sun, H. (2019) Deletion of Clock Gene Bmal1 Impaired the Chondrocyte Function Due to Disruption of the HIF1 $\alpha$-VEGF Signaling Pathway. Cell Cycle, 18, 1473-1489. https://doi.org/10.1080/15384101.2019.1620572

[20] Ding, H., Zhao, J., Liu, H., Wang, J. and Lu, W. (2020) BMAL1 Knockdown Promoted Apoptosis and Reduced Testosterone Secretion in TM3 Leydig Cell Line. Gene, 747, Article ID: 144672. https://doi.org/10.1016/j.gene.2020.144672

[21] Yamakawa, T., Matsumoto, T., Hofmann, T. and Kodera Y. (2014) Aged Garlic Extract and One of the Constituent, (+)-(2S,3R)-Dehydrodiconiferyl Alcohol, Inhibits Alkaline Phosphatase Activity Induced by Inflammation Factors in Human Vascular Smooth Muscle Cells. Food and Nutrition Sciences, 5, 177-184. https://doi.org/10.4236/fns.2014.52023

[22] Akita, K., Isoda, K., Sato-Okabayashi, Y., Kadoguchi, T., Kitamura, K., Ohtomo, F. and Daida, H. (2017) An Interleukin-6 Receptor Antibody Suppresses Atherosclerosis in Atherogenic Mice. Frontiers in Cardiovascular Medicine, 4, Article No. 84. https://doi.org/10.3389/fcvm.2017.00084

[23] Ge, G.F., Shi, W.W., Yu, C.H., Jin, X.Y., Zhang, H.H., Zhang, W.Y. and Yu, B. (2017) Baicalein Attenuates Vinorelbine-Induced Vascular Endothelial Cell Injury and Chemotherapeutic Phlebitis in Rabbits. Toxicology and Applied Pharmacology, 318, 23-32. https://doi.org/10.1016/j.taap.2017.01.013

[24] López-Mejías, R. and González-Gay, M.A. (2019) IL-6: Linking Chronic Inflammation and Vascular Calcification. Nature Reviews. Rheumatology, 15, 457-459. https://doi.org/10.1038/s41584-019-0259-x

[25] Li, C., He, J., Zhong, X., Gan, H. and Xia, Y. (2018) CX3CL1/CX3CR1 Axis Contributes to Angiotensin II-Induced Vascular Smooth Muscle Cell Proliferation and Inflammatory Cytokine Production. Inflammation, 41, 824-834. https://doi.org/10.1007/s10753-018-0736-4

[26] Zhao, J., Niu, X., Yu, J., Xiao, X., Li, W., Zang, L. and Li, W. (2020) Poria cocos Polysaccharides Attenuated Ox-LDL-Induced Inflammation and Oxidative Stress via ERK Activated Nrf2/HO-1 Signaling Pathway and Inhibited Foam Cell Formation in VSMCs. International Immunopharmacology, 80, Article ID: 106173. https://doi.org/10.1016/j.intimp.2019.106173

[27] Zha, M., Tian, T., Xu, W., Liu, S., Jia, J., Wang, L. and Huang, L. (2020) The Circadian Clock Gene Bmal1 Facilitates Cisplatin-Induced Renal Injury and Hepatization. Cell Death and Disease, 11, Article No. 446. https://doi.org/10.1038/s41419-020-2655-1

[28] Ingle, K.A., Kain, V., Goel, M., Prabhu, S.D., Young, M.E. and Halade, G.V. (2015) Cardiomyocyte-Specific Bmal1 Deletion in Mice Triggers Diastolic Dysfunction, Extra- 
cellular Matrix Response, and Impaired Resolution of Inflammation. American Journal of Physiology. Heart and Circulatory Physiology, 309, H1827-H1836.

https://doi.org/10.1152/ajpheart.00608.2015

[29] Early, J.O., Menon, D., Wyse, C.A., Cervantes-Silva, M.P., Zaslona, Z., Carroll, R.G. and Curtis, A.M. (2018) Circadian Clock Protein BMAL1 Regulates IL-1 $\beta$ in Macrophages via NRF2. Proceedings of the National Academy of Sciences of the United States of America, 115, E8460-E8468. https://doi.org/10.1073/pnas.1800431115

[30] Oishi, Y., Hayashi, S., Isagawa, T., Oshima, M., Iwama, A., Shimba, S. and Manabe, I. (2017) Bmal1 Regulates Inflammatory Responses in macrophages by Modulating Enhancer RNA Transcription. Scientific Reports, 7, Article No. 7086.

https://doi.org/10.1038/s41598-017-07100-3

[31] Sugimoto, T., Morioka, N., Zhang, F.F., Sato, K., Abe, H., Hisaoka-Nakashima, K. and Nakata, Y. (2014) Clock Gene Per1 Regulates the Production of CCL2 and Interleukin-6 through p38, JNK1 and NF- $\mathrm{KB}$ Activation in Spinal Astrocytes. Molecular and Cellular Neurosciences, 59, 37-46. https://doi.org/10.1016/j.mcn.2014.01.003

[32] Kobayashi, E.H., Suzuki, T., Funayama, R., Nagashima, T., Hayashi, M., Sekine, H. and Yamamoto, M. (2016) Nrf2 Suppresses Macrophage Inflammatory Response by Blocking Proinflammatory Cytokine Transcription. Nature Communications, 7, Article ID: 11624 . https://doi.org/10.1038/ncomms11624

[33] Aravani, D., Foote, K., Figg, N., Finigan, A., Uryga, A., Clarke, M. and Bennett, M. (2020) Cytokine Regulation of Apoptosis-Induced Apoptosis and Apoptosis-Induced Cell Proliferation in Vascular Smooth Muscle Cells. Apoptosis, 25, 648-662.

https://doi.org/10.1007/s10495-020-01622-4

[34] Reustle, A. and Torzewski, M. (2018) Role of p38 MAPK in Atherosclerosis and Aortic Valve Sclerosis. International Journal of Molecular Sciences, 19, Article No. 3761. https://doi.org/10.3390/ijms19123761

[35] Kyaw, M., Yoshizumi, M., Tsuchiya, K., Kirima, K. and Tamaki, T. (2001) Antioxidants Inhibit JNK and p38 MAPK Activation but Not ERK 1/2 Activation by Angiotensin II in Rat Aortic Smooth Muscle Cells. Hypertension Research, 24, 251-261. https://doi.org/10.1291/hypres.24.251

[36] Wang, Z., Castresana, M.R. and Newman, W.H. (2004) Reactive Oxygen Species-Sensitive p38 MAPK Controls Thrombin-Induced Migration of Vascular Smooth Muscle Cells. Journal of Molecular and Cellular Cardiology, 36, 49-56. https://doi.org/10.1016/j.yjmcc.2003.09.014

[37] Hilenski, L.L., Clempus, R.E., Quinn, M.T., Lambeth, J.D. and Griendling, K.K. (2004) Distinct Subcellular Localizations of Nox1 and Nox4 in Vascular Smooth Muscle Cells. Arteriosclerosis, Thrombosis, and Vascular Biology, 24, 677-683. https://doi.org/10.1161/01.ATV.0000112024.13727.2c

[38] Liu, X., Guo, Y., Yang, Y., Qi, C., Xiong, T., Chen, Y. and Wang, D. (2021) DRD4 (Dopamine D4 Receptor) Mitigate Abdominal Aortic Aneurysm via Decreasing p38 MAPK (Mitogen-Activated Protein Kinase)/NOX4 (NADPH Oxidase 4) Axis-Associated Oxidative Stress. Hypertension, 78, 294-307. https://doi.org/10.1161/HYPERTENSIONAHA.120.16738

[39] Goldsmith, C.S. and Bell-Pedersen, D. (2013) Chapter One-Diverse Roles for MAPK Signaling in Circadian Clocks. Advances in Genetics, 84, 1-39. https://doi.org/10.1016/B978-0-12-407703-4.00001-3

[40] de Paula, R.M., Lamb, T.M., Bennett, L. and Bell-Pedersen, D. (2008) A Connection between MAPK Pathways and Circadian Clocks. Cell Cycle, 7, 2630-2634. 
https://doi.org/10.4161/cc.7.17.6516

[41] Petrzilka, S., Taraborrelli, C., Cavadini, G., Fontana, A. and Birchler, T. (2009) Clock Gene Modulation by TNF-Alpha Depends on Calcium and p38 MAP Kinase Signaling. Journal of Biological Rhythms, 24, 283-294.

https://doi.org/10.1177/0748730409336579

[42] Kim, C.J., Yoon, B.H., Romero, R., Moon, J.B., Kim, M., Park, S.S. and Chi, J.G. (2001) Umbilical Arteritis and Phlebitis Mark Different Stages of the Fetal Inflammatory Response. American Journal of Obstetrics and Gynecology, 185, 496-500. https//doi.org/10.1067/mob.2001.116689 